\section{Chronische Lungen- erkrankung: Welches Regime schützt besser vor COPD-Rezidiven?}

Wedzicha JA et al. Indacaterol-Glycopyrronium versus Salmeterol-Fluticasone for COPD. N Engl J Med 2016; 374: 2222 - 2234

Die Kombination Salmeterol/Fluticason bewahrt Patienten mit fortgeschrittener COPD effektiver vor Exazerbationsrezidiven. Dies gilt im Vergleich zur Variante Indacaterol/Glycopyrronium, wie die prospektive Multizenterstudie von Jadwiga $A$. Wedzicha und Kollegen zeigt.

Die Leitlinien zur Rezidivprophylaxe bei COPD lassen sowohl die inhalative Anwendung von beta-Agonisten (longacting beta-agonist: LABA), kombiniert mit Kortison, als auch den Einsatz von Muscarinantagonisten (long-acting muscarinic antagonist: LAMA) zu. Noch offen ist in diesem Zusammenhang der Stellenwert einer Kombination LABA/LAMA. Bei der Bewertung dieser Therapieoptionen muss die, auch durch den inhalativen Kortisoneinsatz induzierte, Nebenwirkungsproblematik berücksichtigt werden.

Im Rahmen der FLAME-Multizenterstudie therapierten die Autoren 3362 COPD-Patienten (Stadium GOLD>2) randomisiert mit einer LABA/Kortison-Kombination (Salmeterol/Fluticason) oder einer LABA/ LAMA-Kombination (Indacaterol/Glycopyrronium) für 52 Wochen. Im vorangegangenen Jahr hatte wenigstens eine Exazerbation stattgefunden. Die $\mathrm{FEV}_{1}$ nach Bronchodilatatortest musste um mindestens $25 \%$ vermindert sein. Bei $40 \%$ der Studienteilnehmer bestand ein gegenwärtiger Nikotinabusus.

Die Autoren erfassten alle im Studienzeitraum aufgetretenen COPD-Exazerbationen und klassifizierten diese entsprechend des Schweregrads. Für 30 Tage nach Beendigung der Studie wurde weiter nachbeobachtet. Neben der Exazerbationsrate, als primärem Studienziel, dokumentierten die Forscher auch die Latenz bis zur ersten Exazerbation. Auch mögliche, klinische Kofaktoren berücksichtigten die Forscher.

Die Auswertung zeigte eine, unter Indacaterol/Glycopyrronium verminderte, Exazerbationsrate (3,59 vs. 4,03/Jahr) sowie einen verlängerten Zeitraum bis zum ersten Exarzerbationsereignis (71 vs. 51 Tage). Die errechnete Rate der mäßigen bis schweren Exazerbationen unter Indacaterol/Glycopyrronium war ebenfalls reduziert (0,98 vs. $1,19 /$ Jahr). Der günstige Effekt der Indacaterol/GlycopyrroniumKombination zeigte sich unabhängig von klinischen Kofaktoren, wie etwa einer Eosinophilie.

Bei der globalen Nebenwirkungsrate ergab sich kein gruppenspezifischer Befund (gravierende Nebenwirkungen ca. $19 \%)$. Auffallend war allerdings die, unter der Kombination Indacaterol/Glycopyrronium verminderte, Pneumonieinzidenz (3,2\% vs. 4,8\%). Die Mortalität in beiden Therapiegruppen lag bei 1,4\%.

FAZIT

Patienten mit fortgeschrittener COPD profitieren von einer Kombinationsprophylaxe mit Indacaterol/Glycopyrronium. Im Vergleich mit Salmeterol/ Fluticason zeigt sich eine reduzierte Exazerbationsrate bei günstigem Einfluss auf die Nebenwirkungslast.

Dr. med. Horst Gross, Berlin 